\title{
DAS INTERNATIONALE CIPAC-VERZEICHNIS IM 15. JAHR SEINES BESTEHENS
}

\section{von Otto Oberhauser}

Die Webseite The International CIPAC List (http://cipacs.vfi-online.org/) besteht seit 2001. Sie verzeichnet „card-image public access catalogues“ (ClPACs), also Online-Kataloge auf der Basis digitalisierter (gescannter) Zettel- bzw. Bandkataloge. Für Fernleihabteilungen sowie für wissenschaftlich Tätige, die an älterer Literatur interessiert sind, sind derartige Kataloge oft ein unverzichtbares Hilfsmittel, da sich viele Bibliotheken bisher nicht in der Lage sahen, ihre alten Kataloge einer „richtigen“ Retrokonversion zu unterziehen. Sie griffen daher zu der vergleichsweise günstigen Alternative, die Katalogkarten zu scannen und mit Hilfe einer geeigneten Software im Web zur Suche anzubieten.

Das erste bekannte Beispiel eines solchen Katalogs war der 1994 realisierte „Electronic Card Catalog“ der Universitätsbibliothek Princeton, NJ (USA). Interessanterweise wird er trotz der dort inzwischen durchgeführten vollständigen Katalogkonversion immer noch im Web angeboten - vermutlich aufWunsch der Benutzerschaft. Katalogkarten, so sagt man, seien mitunter doch etwas ganz anderes als Datensätze in Online-Katalogen, man denke etwa an handschriftliche Annotationen, typographische Besonderheiten oder Beschreibungsdetails, die bei Anwendung neuerer Regelwerke entfallen.

Seit der Mitte der 1990er Jahre begannen sich CIPACs auch in Europa zu etablieren. Das zunächst als Nebenprodukt meiner Birminghamer MasterArbeit (2002) erstellte Verzeichnis aller zu jener Zeit bekannten Kataloge dieser Art begann 2001 mit etwa 20 Links. Aufgrund des damals bloss vermuteten Interesses an einem solchen Verzeichnis wurde es im Web behalten und weiter gepflegt. Rasch wuchs es auch an: Anfang 2002 waren es bereits 50 Kataloge aus 11 Ländern, Anfang 2007 gar schon 110 Kataloge aus 19 Ländern. Nunmehr, im fünfzehnten Jahr seines Bestehens, umfasst das Verzeichnis rund 170 Einträge zu CIPACs in 31 Ländern (Stand: Februar 2015). Die Angabe „rund“ soll ausdrücken, dass nicht immer eindeutig ist, was genau ein Eintrag ist, da es in einigen Fällen mehrere Links zu einer Bibliothek gibt. In anderen und deutlich mehr Fällen führt der (als nur ein Eintrag gezählte) Link zu einer Bibliothek dort zu mehreren CIPACs, die auf einer Sammelseite gelistet sind. Auf den ersten Blick verzeichnet Deutschland das stärkste Kontingent, doch bei genauerer Betrachtung hält Italien die Spitze, 
da eine der sieben aus diesem Land angeführten Adressen zu einer Webseite führt, die 219 historische Kataloge aus 37 italienischen Bibliotheken nachweist. Stark vertreten sind auch Österreich und die Tschechische Republik. Zu den jüngst hinzugekommen Ländern zählen etwa Kuba und Albanien.

Im Jahr 2007 wurde das Verzeichnis in eine neue Form gebracht, in der pro angezeigter Seite immer nur die Kataloge des jeweils zuvor selektierten Landes aufgelistet werden. Dadurch wurde das zuvor nötige Scrollen weitgehend vermieden. Lediglich die Auflistung für Deutschland umfasst auf den meisten Bildschirmen mehr als eine Seite. Aufgrund des Anwachsens der Länderliste muss inzwischen auch diese auf Standardbildschirmen bereits gescrollt werden.

Das vor 15 Jahren von meinem Betreuer in Birmingham, Denis F. Reardon, in Analogie zu OPACs vorgeschlagene Akronym CIPACs, das in meinen Publikationen sowie im gegenständlichen Webverzeichnis weiter propagiert wurde, hat sich bedauerlicherweise nicht vollständig durchgesetzt. Obwohl es durchaus häufig verwendet wird, wird in Deutschland des öfteren die Alternative IPACs vorgezogen, die uns damals wie heute zu unspezifisch erschien, da vieles ein Imagekatalog sein kann - nicht nur ein Katalog auf der Basis gescannter Katalogkarten. Wenn man nach CIPAC oder CIPACs googelt, sieht man allerdings, dass unser Akronym auch für viele andere Bedeutungen stehen kann (meist Institutionen). Erfreulich ist jedoch, dass bei der Recherche nach „cipacs“ das Verzeichnis an der ersten Stelle der Ergebnisliste aufscheint.

Interessant ist auch, dass die Google-Recherche nach der Webadresse des Verzeichnisses inzwischen über 6.500 Treffer erbringt. Die internationale CIPAC-Liste ist somit auf sehr vielen Webseiten verlinkt und wird wohl auch häufig genutzt. Sie ist zu einem Service geworden, das weiterhin gepflegt und gewartet werden will. Hinweise auf neue bzw. noch nicht verzeichnete $\mathrm{Cl}$ PACs sind daher stets willkommen, ebenso Hinweise auf geänderte Bezeichnungen oder geänderte Webadressen. Zum Wartungsbedarf zählt aber auch das Entfernen von Links, etwa wenn CIPACs aufgrund von Katalogkonversionen vom Web genommen werden. Die meisten neuen Einträge wie auch Aktualisierungen von Webadressen usw. beruhen allerdings nach wie vor auf eigenen Recherchen. Ausnahmen waren die hilfreichen Mitteilungen einiger weniger Kollegen, in jüngerer Vergangenheit etwa Ulrich Schaefer (Frankfurt am Main), Lothar Karl (München), Aleš Brožek (Ústí nad Labem) und Pedro Urra (Havanna), denen für ihre Informationen ausdrücklich gedankt sei.

Dr. Otto Oberhauser, Wien E-Mail: oberh@web.de 